\title{
Mediación pedagógica: Clave de una educación humanizante y transformadora. Una mirada desde la estética y la comunicación
}

\author{
Pedagogical Mediation: Key to Humanizing and Transformative Education. A Look From \\ Aesthetics and Communication
}

\section{Mediação pedagógica: Chave de uma educação humana e transformadora. Uma ótica a partir da estética e da comunicação}

\author{
Faber Andrés Alzate-Ortiz \\ Corporación Universitaria Minuto de Dios \\ Grupo de Estudios e Investigaciones Educativas y Pedagógicas \\ Bello, Colombia \\ faber.alzate@uniminuto.edu \\ https://orcid.org/0000-0002-3102-7666 \\ Juan Carlos Castañeda-Patiño \\ Universidad del Quindío \\ Grupo de Investigación en Desarrollos Electrónicos y Tecnológicos \\ Armenia, Colombia \\ juanchoca75@hotmail.com \\ https://orcid.org/0000-0002-7920-4588
}

Recibido • Received • Recebido: 20 / 02 / 2018

Corregido • Revised • Revisado: 06 / 08 / 2019

Aceptado • Accepted • Aprovado: 07/ 11 / 2019

\begin{abstract}
Resumen: El presente ensayo propone un análisis epistémico alrededor de la mediación pedagógica partiendo de diferentes elementos teórico-prácticos relacionados con la educación, la comunicación y la estética desde el horizonte de las intencionalidades del aprendizaje en los procesos de formación humana. Se asume que la mediación, por ella misma, es una práctica que carga de sentido y reconocimiento a las personas en formación y sus contextos, por lo tanto, dispone no solo de estrategias y recursos debidamente seleccionados para promover el desarrollo de las capacidades a través de la apropiación de conocimientos plurales, sino que, además, pretende mejorar aquellas concepciones que sobre didáctica y evaluación tradicionalmente se han tenido. En este orden de ideas, a lo largo del trabajo, será posible reconocer, de manera singular y propositiva, algunas posturas de nivel conceptual que sugieren pensar la educación contemporánea desde el escenario pedagógico de las mediaciones. Se facilita, con ello, que los maestros y maestras puedan re-significar todos aquellos modelos, corrientes y tendencias escolares que han estado basadas en una lógica formativa transmisionista, memorística y lineal, centrada en la enseñanza y poco interesada en el aprendizaje.
\end{abstract}

Palabras claves: Mediación pedagógica; proceso formativo; intencionalidad; enseñanza; aprendizaje; 
doi: http://doi.org/10.15359/ree.24-1.21

URL: http://www.una.ac.cr/educare

CORREO: educare@una.cr

comunicación; educación.

\begin{abstract}
This essay proposes an epistemic analysis around pedagogical mediation based on different theoretical and practical elements related to education, communication, and aesthetics, from the horizon of learning intentions in human formation processes. It is assumed that mediation, in itself, is a practice that carries meaning and recognition to people in training and their contexts. Therefore, not only does mediation have properly selected strategies and resources to promote capacity development through the appropriation of plural knowledge, but it also aims to improve those conceptions that have traditionally been about didactics and evaluation. In this order of ideas, throughout the work, it will be possible to recognize, in a singular and propositive way, some positions of conceptual level that suggest thinking of contemporary education from the pedagogical scenario of mediations, facilitating with it that teachers can re-signify all those models, currents and school tendencies that have been based on a transmissive, memorial, and linear formative logic, focused on teaching and little interested in learning.
\end{abstract}

Keywords: Pedagogical mediation; formative process; intentionality; teaching; learning; communication; education.

Resumo: O presente artigo propõe uma análise epistemológica sobre a mediação pedagógica a partir de diferentes elementos teórico-práticos relacionados à educação, a comunicação e a estética, desde a perspectiva das intencionalidades da aprendizagem nos processos de formação humana. Assume-se que a mediação, por si mesma, é uma prática carregada de sentido e reconhecimento às pessoas em formação e seus contextos, por tanto, dispõe não só de estratégias e recursos devidamente selecionados para promover o desenvolvimento de capacidades através da apropriação de conhecimentos plurais, mas também, pretende melhorar aquelas concepções já existentes sobre didática e avaliação. Nesta ordem de ideias, no processo da pesquisa, será possível reconhecer, de forma singular e propositiva, algumas posturas a nível conceitual que sugerem pensar na educação contemporânea desde o panorama pedagógico das mediações . Isso permite que docentes possam resinificar todos aqueles modelos, correntes e tendências escolares baseadas em uma lógica formativa de transmissão, memorização e linearidade, centrada no ensino e pouco interessada na aprendizagem.

Palavras chave: Mediação pedagógica; processo formativo; intencionalidade; ensino; aprendizagem; comunicação; educação.

\title{
Introducción
}

En muchos de los escenarios educativos la palabra mediación pedagógica puede tener cabida entre los discursos y prácticas de personal docente y directivo, lo que no se puede evidenciar a ciencia cierta es si se está logrando una apropiación epistémica real que permita develar desde este concepto, y desde su mismo propósito, una comprensión estética de la educación que favorezca un sentir, pensar y actuar pedagógico más humanizado y, con ello, un obrar escolar capaz de superar la visión clásica de la educación centrada en los contenidos, en 
las verdades absolutas, en la reglas/normas generalizantes y en una linealidad cartesiana que históricamente ha regulado el currículo.

Por lo tanto, desde lo estético, la mediación pedagógica se convierte en una construcción en clave de desarrollo humano, que exige promover educativamente una red de posibilidades de aprendizaje basada en la interconexión ser humano-mundo, de manera que oportunamente podamos estar de "frente a un pensamiento eminentemente poiético, es decir, productivo y creativo" (Najmanovich; 2005 p. 30). De allí, que la mediación pueda considerarse no solo una intención de pensamiento pedagógico innovador y propositivo, sino un verdadero accionar didáctico que coloque en el centro de las reflexiones docente-aprendizaje-estudiantes el acto comunicativo como principal propósito en el ejercicio de la formación.

Ahora, la educación del presente reclama nuevas/otras maneras de concebiry desarrollar los procesos de enseñanza y aprendizaje al interior de la escuela ${ }^{1}$ y no solo por la marcada influencia que las tecnologías de la información y la comunicación están ejerciendo en niños, niñas, jóvenes y personas adultas a la hora de acceder o construir conocimiento y sus posibilidades, sino por los nuevos desafíos que tiene el planeta entero en preservar su existencia, lo que exige la promoción de una formación humana en todos los niveles, pedagógicamente mediada, que potencie una evolución en la formas de inteligencia capaz de utilizar el dato, la información, el saber no solo para crear nuevos modos de relacionarnos armónicamente ser humano-naturaleza reconfigurando el sentido la vida misma, sino que sea posible inter-mediar entre la visión global y el hacer local de la educación para co-construir una visión educadora planetaria que soporte el proyecto de llegar a ser una sociedad culturalmente más evolucionada y sostenible.

Es en esta línea académica que el presente trabajo exhorta a la reflexión pedagógica en clave de tres preguntas gnoseológicas sugerentes:

- ¿Cuáles son las relaciones educativas emergentes entre lo estético y las mediaciones pedagógicas?

- ¿Cómo situar epistémicamente una educación que potencie lo humano desde el escenario de las mediaciones pedagógicas?

- ¿Qué papel juega la comunicación en los procesos educativos que soportan sus intencionalidades formativas en nuevas experiencias estéticas y desde otros lenguajes?

Las anteriores preguntas constituyen, a manera de faro orientador, las pretensiones epistemológicas que situarán la reflexión investigativa que se tratará a lo largo del artículo, al tiempo que servirán de polo atractor para aterrizar las consideraciones que sobre las categorías centrales se

\footnotetext{
${ }^{1}$ Cuando se haga referencia a la palabra escuela, con ello, se está haciendo alusión a todas las instituciones educativas que ofrecen desde el nivel preescolar hasta el nivel de doctorado.
} 
doi: http://doi.org/10.15359/ree.24-1.21

URL: http://www.una.ac.cr/educare

CORREO: educare@una.cr

irán desarrollando. Sin embargo, los planteamientos aquí propuestos no pueden ser considerados verdades absolutas, sino construcciones epistémicas abiertas que posibiliten académicamente seguir re-de-construyendo los argumentos desde la mirada de otros pensamientos educativos.

\section{Claves epistémicas para entender el concepto de mediación pedagógica desde una visión estética de la educación}

En primer lugar, es importante destacar que la educación es un proceso culturalmente vital, que no restringe, limita o violenta, sino que promueve, emociona, libera y motiva al ser humano para potenciar sus capacidades y superar sus limitaciones, de allí, que los elementos que constituyen el diario vivir en el ámbito escolar deben estar orientados a favorecer procesos académicos que representen una oportunidad para que cada persona se transformey transforme su entorno (Freire, 2011). Para nadie es un secreto que todo lo que sucede en la escuela representa niveles de influencia directa e indirectamente en los procesos de aprendizaje de estudiantes, un asunto que necesariamente requiere ser tenido en cuenta por el personal docente mediador a la hora de concebir pedagógicamente sus procesos de enseñanza. Por tanto, en la actualidad el saber y hacer del profesorado debe re-significarse, de manera que pueda dejar atrás el rol clásico de ser sujeto profesional transmisor/repetidor de contenido/información, con base en estándares obsoletos de comunicación, para lograr una mediación pedagógica de la formación humana (Tébar, 2009).

En este sentido, tal como lo sugieren Gutiérrez y Prieto (1999), los actuales educadores y educadoras tienen un doble reto pedagógico. Por un lado construir puentes entre estudiantes, los conocimientos y el contexto; y, por el otro, dotar de sentido su práctica docente a partir de la mediación pedagógica, un asunto que devela a manera de dominio didáctico comprensión gnoseologica de la educación (Fermozo, 1991), la capacidad de promover la enseñanza desde una pedagogía que se soporta en el aprendizaje; es decir, que no solo devuelve la mirada sobre las personas (sus capacidades cognitivas, corporales y espirituales), el contexto y no exclusivamente sobre los contenidos, sino que además, supera la reflexión-acción disciplinar y avanza hacia procesos educativos inter y transdisciplinarios (Gutiérrez, 2004). Sin embargo, ¿qué hace pedagógica una mediación?, ¿cuál es el rol de quienes participan en la mediación pedagógica?, ¿cómo ser docentes que median con criterios de calidad pedagógica? y ¿cuáles son aquellos componentes que constituyen las estrategias que soportan toda mediación pedagógica? Estas son algunas preguntas que orientan los propósitos epistemológicos que a lo largo de esta reflexión investigativa se intentan develar.

Según Gutiérrez (citado por Prieto, 2017, p. 26), "Ilamamos pedagógica a una mediación capaz de promover y acompañar el aprendizaje, es decir, la tarea de construirse y de apropiarse del mundo y de uno mismo, desde el umbral del otro, sin invadir ni abandonar", un escenario 
de educación que debe leerse en clave de la intencionalidad formativa que no es más que el resultado de un adecuado manejo teórico-práctico de los principios de la pedagogía, como lo son la educabilidad y la enseñabilidad; puesto que al primero le corresponde responder las preguntas relacionadas sobre el sujeto que aprende, mientras al segundo, le compete estudiar todo lo relacionado con el dominio epistemológico de la ciencia que se pretende enseñar. Esto quiere decir que la mediación pedagógica deja de ser, como muchos lo consideran, un simple recurso didáctico, para pasar a constituirse en una acción creativa de cada docente, que requiere comprender integralmente la naturaleza de lo que se desea enseñar y aprender, más las características de las personas involucradas en el proceso formativo y sus realidades contextuales, con el propósito de hacer no solo más pertinente y oportuno el acceso al conocimiento, la practica de habilidades y la exploración afectiva del fenomeno de aprender, sino más significativo y edificante el proceso educativo (Díaz y Hernández, 2005).

La mediación pedagógica comprendida desde este panorama, provoca un quiebre epistemológico en la concepción que clásicamente se tiene sobre la educación, el profesorado, el estudiantado y de la comunidad misma, por forjar interacciones cognitivas, corporales y hasta espirituales que gestan, promueven y cuidan la vida como propósito co-emergente a cualquier proceso educativo que se dé al interior o por fuera de la escuela,, Se eliminan así los roles pedagógicos pasivos para configurar encuentros formativos donde todas las personas son protagonistas y el primer punto de partida para promover la enseñanza y el aprendizaje, es la propia realidad, es la vida misma de los actores que participan del proceso de formación (Castillo y Castillo, 2013).

Desde estos escenarios, la posición pedagógica, didáctica y curricular del maestro o maestra es la de mediación de la cultura, teniendo en cuenta que la mediación es un quehacer de tipo interactivo y estético (Villarruel, 2009) que, por un lado requiere de una actitud diferente basada en un actuar en red, polifórmico, creativo e innovador $y$, por el otro, se convierte en una estrategia para continuar de manera dinámica, ampliando las acciones o situaciones de aprendizaje que el estudiantado recibe o construye con su medio, desde ahí y para este.

En tanto, la cultura como una construcción social, histórica, sistemática y simbólica aporta a lo educativo niveles de interpretación que facilitan la re-construcción de significantes y significados que desde el sentir, pensar y actuar educativo le permiten al personal docente recrear otras estéticas que, en clave de mediación pedagógica, llegan a constituirse en caminos/ rutas/planos diversos y diferenciales que dan sentido a la enseñanza y el aprendizaje en un momento/época determinada. De esta manera, cuando el profesorado materializa en sus mediaciones pedagógicas los significados simbólicos que la sociedad desde la perspectiva que la cultura le ha designado, está logrando por lo menos tres niveles de desarrollo profesional que 
doi: http://doi.org/10.15359/ree.24-1.21

URL: http://www.una.ac.cr/educare

CORREO: educare@una.cr

develan calidad en su quehacer, ellos son: primero, haber alcanzado capacidades psicológicas superiores, segundo, ser mediador del currículo, la evaluación y la didáctica para promover emancipación social y, tercero, estar transformando permanentemente sus prácticas en experiencias de vida que le dan sentido a cada docente y a las demás personas (Pérez, Molina, Hernández, Rojas y Murillo, 1991).

La mediación pedagógica, como ya se ha destacado en los planteamientos anteriores, desde la naturaleza teórico-práctica de sus intencionalidades hace una apuesta integral para que la calidad en los procesos de formación represente culturalmente un avance para las comunidades y sus individuos, en la medida en que lleva a la escuela a superar una educación centrada en el contenido y la conduce, más bien, a construir proyectos educativos inspirados en una visión ecológica y humanizante de la pedagogía, un reto que pone de manifiesto un nuevo rol del personal educador planetario a partir del cual se hace necesario cultivar cualidades tales

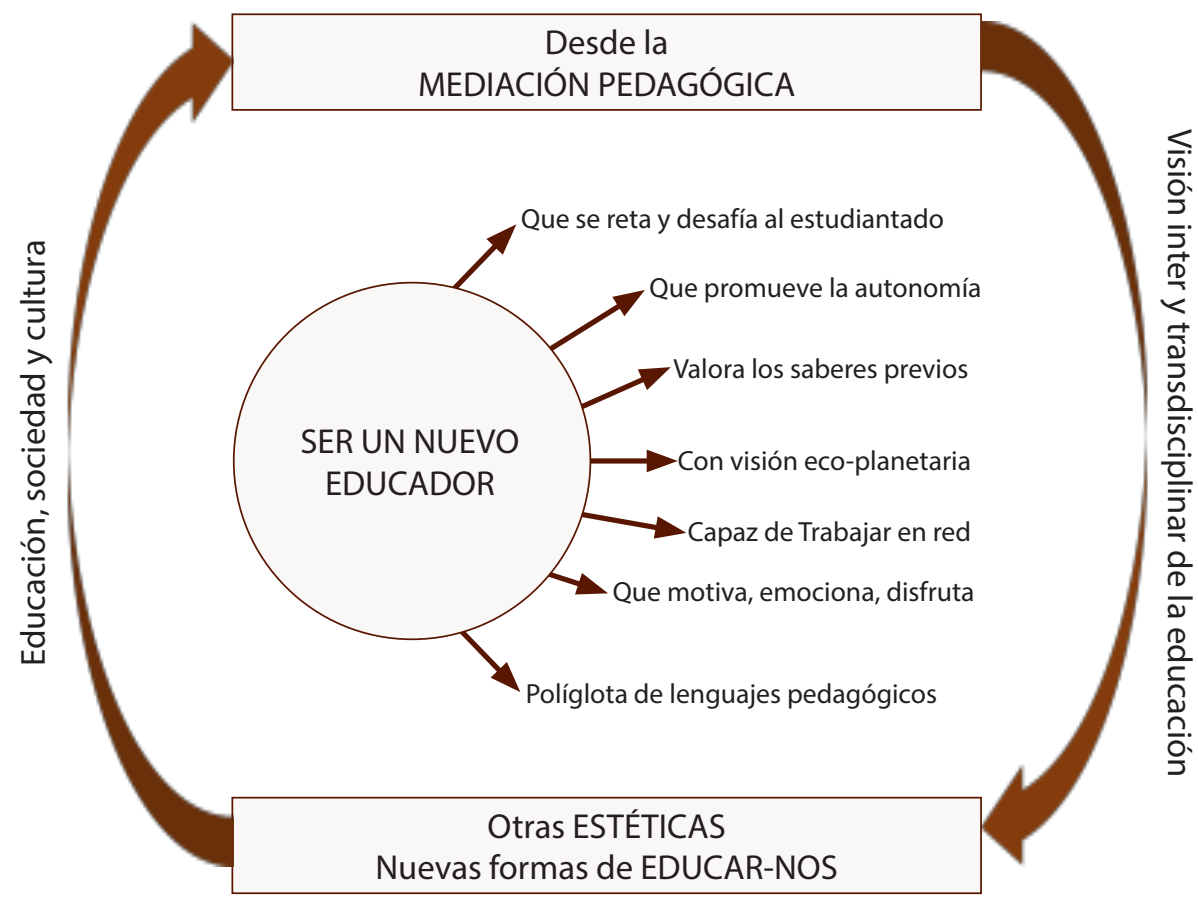

Figura 1: Retos del educador desde la mediación pedagógica.

Nota: Elaboración propia.

como: dominio de la disciplina que se enseña, capacidad para crear e implementar estrategias pertinentes, disposición para democratizar los procesos de enseñanza y aprendizaje, habilidad para valorar y utilizar la realidad, experiencias, vivencias de estudiantes, talento potencial para lograr altos niveles de metacognición entre sus aprendientes, interés por construir y presentar desafíos que lleven a estudiantes a sentir placer por aprender y destreza para estimular la autogestión, la 
autorregulación y la autoevaluación (Assmann, 2002; Labarrere, 2003). (Ver Figura 1).

Como se puede observar en el gráfico anterior, es desde el plano de la mediación pedagógica donde se crea una visión inter y transdisciplinar por parte del personal educador sobre los procesos de enseñanza y aprendizaje y, desde allí, se gestan otras formas estéticas de generar, evolucionar, gestar, reconfigurar, transferir y facilitar la utilización del conocimiento al tiempo que se promueven nuevas formas de educación en las que nos implicamos e implicamos a las otras personas y lo otro; adicionalmente, de manera recursiva y pasando por la triada educación, sociedad y cultura, es posible no solo diseñar e implementar las mediaciones pedagógicas, sino también valorarlas en clave de mejoramiento continuo. Esta valoración debe tener como base por lo menos cinco componentes que soporten las estrategias de la mediación, los cuales son:

- El psicológico, desde el cual se tienen en cuenta los elementos como la motivación, la metacognición, el aprendizaje significativo y la capacidad para comunicar pertinentemente. Estos y otros aspectos aperturan otras comprensiones desde las neurociencias para generar sentido a la misma evolución del cerebro de la especie Sapiens Sapiens, en alusión a la condición intelectual y comportamental del ser humano moderno, como sujeto que piensa, razona y se hace consciente.

- El filosófico, compromete las posibilidades de promover la reflexión educativa crítica y abierta, en torno al aprendizaje como centro de las problematizaciones del conocimiento pedagógico que puede realizar el personal docente, a partir de su accionar curricular y, con ello, cultivar su espíritu de investigador escolar.

- El político, desde donde se privilegia la acción creadora del educar y el comunicar para emanciparse y emancipar al estudiantado desde el escenario mismo de la formación, de manera que juntos adquieran las habilidades para formar una ciudadanía libre, participativa, democrática y respetuosa del bien común.

- El pedagógico, para consolidar una visión inter y transdisciplinaria de la enseñanza y el aprendizaje desde la lógica de la comunicabilidad y la estética como entes facilitadores de los procesos educacionales contemporáneos, teniendo en cuenta la esencia biológica del bienestar natural de los individuos que llegan a ser protagonistas en todo proceso de mediación.

- El tecnológico, el que permite gestar la mediación a partir de la compresión y uso pedagógico de los medios, los cuales reúnen un sin número de herramientas que le permiten al personal docente dinamizar los procesos de enseñanza y aprendizaje a través del mejoramiento de los canales comunicativos y la implementación de otras estéticas educativas más atractivas para el estudiantado.

Finalmente, los anteriores componentes son importantes a la hora de consolidar las estrategias que soportarán toda mediación pedagógica, de manera que se logre hacer 
doi: http://doi.org/10.15359/ree.24-1.21

URL: http://www.una.ac.cr/educare

CORREO: educare@una.cr

evidente la intencionalidad de los procesos formativos en clave de aprendizaje $y$, para ello, se hace necesario consolidar, de forma interdisciplinar, diferentes relaciones epistémicas como la psicológico-pedagógica, la político-filosófica, la tecnológica-pedagógica-psicológica, entre otras, que faciliten una comprensión más pertinente, integral, holística y oportuna de los procesos educativos, al tiempo que permita superar, desde lo pedagógico, lo didáctico y lo evaluativo, las concepciones teórico-prácticas que se tienen sobre la enseñanza.

\section{Planos de actuación escolar entre la experiencia estética y las vivencias pedagógicas en el seno de una educación humanizante y humanizada}

El bienestar propio de la experiencia estética es parte de este bienestar natural. En realidad, creo que una vida humana vivida en el bienestar natural como lo he definido, es una vida humana vivida en una total estética en las muchas dimensiones de la vida. (Maturana, 1995, p. 58)

Por la imaginación, el hombre y la mujer pueden hacer más vivible y esperanzadora la vida humana. Por el rompimiento de las ataduras derivadas de la ciencia mecanicista: puede ir más allá de lo establecido y normalizado; pueden traspasar fronteras y explorar nuevas posibilidades creando y recreando nuevas interacciones e interrelaciones. (Gutiérrez y Prado, 2015, p. 97)

El acto del educar-nos debe suponer, en primer orden, un escenario vital de bienestar individual y colectivo, a partir de diversas experiencias estéticas multidimensionales en donde sea posible superar aquellas lógicas formativas basadas en un saber pedagógico lineal y absolutista que suele estar soportado en una visión científica mecanicista del mundo educativo en donde lo importante no siempre resulta ser la persona sino los contenidos. En sintonía con los autores arriba mencionados, es urgente cargar de sentido ecológico y humano los procesos de formación que se promuevan en los entornos educativos, en clave de las diversas posibilidades de interacción que pueden darse entre aprendientes, contexto y saberes científicos y socioculturales que se promueven desde los currículos.

En este orden de ideas, humanizar la educación es un reto ineludible para los hombres y las mujeres de nuestros tiempos, porque nos han invitado a hacer de nuevo la tarea pedagógica, ya no para repetir contenidos y dicotomizar las humanas experiencias de aprendizaje basadas en las limitadas verdades absolutas, únicas y endiosadas a lo largo de nuestra historia, sino para embellecer el encuentro con los niños, niñas, jóvenes y personas adultas desde otras/ nuevas educaciones que realmente estén cimentadas en el placer/bienestar de existir en todas las dimensiones de la vida, para que sea posible recrear la experiencia del ser y, con ello, el saber desde múltiples miradas, múltiples encuentros, diversos entornos y cosmovisiones educulturales. 
En este sentido, Maturana (1995) nos hace una buena exhortación, al insistir en que debemos entrar en armonía con la vida, con lo vivido, dejando atrás los deseos, las emociones, los intereses incompatibles, toda vez que destruyen la coherencia del flujo de la vida y generan experiencia de fealdad, por lo que la educación está llamada a embellecerse propiciando espacios de encuentro humano donde sea posible vivir en armonía, sincronicidad, empatía, amor, ternura y sana convivencia para que se generen recursivamente experiencias estéticas de aprendizaje vital.

En otras palabras, es importante considerar que ha sido el ingenio imperioso y dominante de una parte de la raza humana que, a lo largo de muchos años, ha basado sus decisiones en el criterio de utilidad y control, el que ha creado condiciones para legitimar desde la dualidad, lo cartesiano, lo lineal y lo simplista, lo supuestamente "importante" en la educación de la condición humana, que es colocarle límites y cánones a las mismas experiencias de aprendizaje, con el fin de entorpecer su desarrollo integral. Al imponer paradigmas, enfoques y modelos se propician contradicciones y situaciones de crisis en el yo espiritual, mental y corporal de las personas, lo que lleva a, finalmente, obedecer y pensar condicionados y a crear según sea el valor de uso. De acuerdo con Hathaway y Boff (2014):

La vía que conduce a la luz parece oscura, la senda que va hacia adelante parece ir hacia atrás, el camino derecho parece tortuoso, el mayor poder parece débil, la más pura virtud parece mancillada, la auténtica abundancia parece insuficiente, la genuina firmeza parece inestable. El espacio más vasto no puede ser contenido, el más grande talento tarda en madurar, la nota más alta es difícil de oír, no puede darse concreción a la forma más perfecta. (p. 27)

En esta postura, encontramos elementos de mediación pedagógica para abordar esos asuntos que inquietan el sentir, pensar y hacer educativo, al considerar al igual que Hathaway y Boff (2014) que la encrucijada que deviene de las dinámicas humanas y sus co-relaciones con la historia de la humanidad y el "oikos" ", la agudización de las pobrezas, degradación ecológica y la destrucción de la misma especie están asociadas con el afán depredador que les genera la urgencia de sabiduría y capacidad para direccionar su propia vibración o energía sin importarles la de su prójimo(s) y, con ello, se diluye, sin duda, la experiencia estética.

Una estética y una lógica que parten de una concepción paradójica admiten en su seno al tiempo, al cambio, a la transformación porque el punto de partida es el de la dinámica vincular y no el de las esencias absolutas del dualismo o el monismo propios de nuestra tradición occidental. (Najmanovich, 2005, p. 26)

Lo anterior nos hace suponer que, al parecer, la humanidad se ha empeñado en morir estando viva, quizás por el terror a la muerte como enuncia Becker (2003), o quizás por creer

\footnotetext{
${ }^{2}$ Expresión griega que significa casa.
} 
doi: http://doi.org/10.15359/ree.24-1.21

URL: http://www.una.ac.cr/educare

CORREO: educare@una.cr

que sus realidades de vida no son propias sino que fueron compradas o expropiadas, por lo que finalmente terminan limitando su acción-comprensión estética de su mundo y del mundo que lo circunda como asuntos que están directamente correlacionados con lo bio-psico-socio-cultural de la condición humana y la educación. Es decir, en un mundo de competencia desmedida basado en la utilidad, la vida se vuelve invivible, porque el heroísmo que ha de adoptar toda persona que desee vivir, debe vencer sus necesidades basadas en el modelo de consumo, para adquirir el derecho a vivir.

Derivado de lo anterior, los sistemas de creencias o de valor de uso, según Hinkelammert y Mora (2012), nos han conducido a la necesidad de luchar por la vida, como un reto o prueba que debe ser superada, asunto que nos ciega frente a la posibilidad de ver la verdad que se manifiesta como conciencia estética, aunque de ello no se percate la mente, que sería tal como aquello creado por el inconsciente colectivo, ya mencionado por Jung, (1991) y Maturana (1995), en donde se terminan delineados o graficados educativamente los senderos por donde ha de dirigirse la vida en función de los acuerdos socialmente instituidos y legitimados culturalmente, como por ejemplo, el patriarcalismo.

Al respecto, mediar pedagógicamente con el objetivo de propiciar la resignificación y movilización/enacción del pensamiento educativo para que emerjan otras posibilidades estéticas de conocer y generar experiencias de aprendizaje vital, propone integrarnos al mundo de la educación desde el contexto de la comunicación, en donde es necesario situar nuevamente al ser humano en función de sus emociones y lenguajes, que vibre en conversaciones críticas y abiertas donde sea posible, indispensable e inevitable "mirar con ojos nuevos" Najmanovich (2005), de manera que se logren reconfigurar las redes, los tejidos, los territorios o cartografías del conocimiento que circunda en torno al hermoso acto de educar. Ahora, según Maturana (1995), "conforme nuestras experiencias estéticas humanas surgen como distinciones que hacemos en conversaciones, las distinciones que hacemos en el dominio de nuestras experiencias estéticas participan en nuestras reflexiones acerca de ellas" (p. 59).

En otras palabras, sería urgente y necesario reconocer en la complejidad que significa la habitancia del mundo, las múltiples perspectivas en el sentir, pensar y hacer de cada una de las personas y sus relaciones con el otro ser y lo otro, mediado por la oportunidad de lenguajear y con ello, de tejer escenarios de comunicación vital que impulsen la posibilidad de desarrollar/ evolucionar nuestro cerebro triuno, en tanto, que nos representa como ideologías, emociones y manifestaciones humanas para que, a su vez, permitan co-crear-nos y autoorganizar-nos en el acto educativo, a partir de diversos significados y significantes, que impulsen otras alternativas de experiencia estética, en donde la ciudadanía planetaria pueda revelar como realidad vivida y vivible nuevas maneras de generación y socialización de conocimientos, al tiempo que también gesta otros modos culturalmente válidos de interacción/coexistencia humana y ecológica. 
Por lo tanto, en sintonía creativa con Najmanovich (2005), se requiere, desde la mediación pedagógica, "una mirada implicada y responsable, sensible y afectiva a la par que inteligente" (pp. 26-27), donde sea posible reconfigurar una comprensión biológicamente diferente de la estética, con prácticas educativas que provoquen experiencias de vida sensibles, emotivas, revitalizantes y interrelacionadas, con el ánimo y la intención epistemica de ser capaces de construir territorios escolares basados en el amor, de manera que se pueda disfrutar, sentir, valorar, experimentar la vida antes que morir viviendo y sin sentido.

Es prudente advertir, entonces, tal como menciona Guardian (1985, citado por Sheldrake, 1981), cuando se refiere a las limitaciones físicas de la experiencia:

Existe una diferencia radical entre la biología del cerebro y la experiencia mental (una experiencia subjetiva a la que los filósofos de la mente denominan qualia), que incluye la experiencia de cualidades como, por ejemplo, "rojo". Chalmers afirma que, si queremos tomarnos en serio la conciencia, es necesario ir más allá del marco de referencia estrictamente materialista. (p. 26)

Insistimos, por lo tanto, en que las mediaciones pedagógicas para comunicar nuevos/ otros referentes, saberes y prácticas acerca de la experiencia estética del educar, tal como lo plantea Maturana (1995), son esencialmente creaciones desde psique y en ella, de manera urgente para la re-significación, ampliación y movilización hacia alternativas/posturas intencionadas didácticas, curriculares, evaluativas e investigativas desde la complejidad misma de la educación como proyecto socio-cultural; por supuesto, en el cual debe existir la suficiente amplitud como para aceptar que somos habitantes de un gran territorio y que debemos actuar en red de manera que no se limite la experiencia y la práctica humana en cualquiera de las dimensiones del desarrollo (Labarrere, 2006).

Finalmente, es oportuno recurrir nuevamente a Najmanovich (2008), al considerar que los nuevos/otros conceptos, re-semantizaciones y re-significaciones en educación y pedagogía pueden seducir a maestros y maestras desde ejercicios profundos de comunicabilidad educativa, si se posibilitara el surgimiento de otros "modos de experienciar el mundo y producir significado. "'Sólo la diferencia puede producir diferencia'. La novedad sólo nace de un encuentro con el otro (interno o externo)" (p. 140). Por lo tanto, la humanización de la educación exige ampliar nuestras comprensiones sobre las formas/maneras pedagógicamente posibles de provocar y potenciar el aprendizaje en los seres humanos, que en otras palabras sería el estudio y vivencia de la cognición estética como una epistemología emergente en las ciencias de la educación.

\section{A manera de reflexiones finales}


doi: http://doi.org/10.15359/ree.24-1.21

URL: http://www.una.ac.cr/educare

CORREO: educare@una.cr

Tomando como referente los diferentes planteamientos descritos a lo largo de la presente reflexión investigativa, es posible afirmar que la mediación pedagógica debe ser concebida, desarrollada y evaluada de manera holística y compleja-en red, soportada en una educación que rescata una visión de la formación basada en lo humano y lo ecológico (Alvarado, 2007), ya que la propuesta de mediación que se espera desarrolle el profesorado mediador deberá estar orientada a promover procesos estéticos de aprendizaje desde vida y para la vida, donde la motivación, el goce, la pasión, la creatividad y la innovación sean pilares en su construcción.

Por lo tanto, la mediación debe concebir de manera integral y equilibrada el contenido, el aprendizaje y la forma a la hora de diseñarse, ejecutarse y evaluarse (Gutiérrez y Prieto, 1999). Esto implica tres retos de orden pedagógico que todo persona educadora deberá plantearse:

1. Hacer de los procesos formativos mediados experiencias estéticas de aprendizaje que sean atractivas y que, sin desdibujar el rigor de los dominios científicos, puedan motivar al estudiantado para que disfrute el acceso al conocimiento y con ello, desee aprender permanentemente.

2. El personal educador mediador debe constituirse en un pedagogo o pedagoga constructivista capaz de articular una educación activa, basada en la experiencia real y contextual, con los avances de la sociedad en materia tecnológica, económica y política, ya que la mediación pedagógica requiere de la participación de sus actores y de los escenarios de despliegue en que se desenvuelven o esperan desenvolverse personal y profesionalmente (Pérez, 2009).

3. La educación mediada pedagógicamente propende por el desarrollo de capacidades superiores que permitan tanto a docentes como a estudiantes consolidar procesos de comunicación asertiva y ecléctica, que faciliten procesos de autoevaluación, coevaluación y heteroevaluación a partir de los cuales sea posible potenciar el pensamiento crítico, flexible y abierto.

4. La mediación comunicada y comunicativa de altenativas para aprender deberá responder de manera tácita y expresa a la epistemología y la gnoseología de la educación, tanto mayor o igual a los asuntos científicos que configuran el conocimiento para la evolución de la humanidad; de tal manera y derivado de lo anterior, debiera surgir la interdisciplinariedad y, por supuesto, la integración con el resto de las ciencias, ya que son esas y otras características las que confieren la complejidad a la misma humanidad.

En este sentido, se hace necesario promover en la escuela mediaciones pedagógicas que propendan por la construcción de prácticas educativas que dejen atrás el paradigma clásico de la transmisión, el control y la sumisión académica y favorezcan, más bien, experiencias de aprendizaje basadas en el diálogo creativo, respetuoso y motivador, la construcción de acciones 
que reten la inteligencia para crear soluciones pertinentes a las necesidades de las comunidades y la promoción de una conciencia estética y ecológica que rescate la condición humana en todas sus dimensiones (Maturana, 2002). En otras palabras, se requiere mediar desde un quehacer pedagógico que potencie el intercambio de argumentos/motivos que impulsen el análisis, la problematización, la de-construcción, la comprehensión, el pensamiento complejo, la crítica constructiva y la consolidación de proyectos escolares a partir de los cuales sea posible intercambiar retos, visiones y acciones tan necesarias para el mundo de hoy, porque en palabras de Gutiérrez y Prado (2015):

Si queremos dar sentido a lo que hacemos, ante todo debemos sentirlo y [sentirlo con nuestros sentidos]. Es evidente, en consecuencia, que el sentimiento, la intuición, la emoción, la vivencia y la experiencia son el norte que nos guiará ilusionados en el construir futuro desde la realidad de cada día. (p. 57)

\section{Referencias}

Alvarado, E. (2007). Educación, ecología y economía: Pasos hacia otra epistemología. San José, Costa Rica: Alma Mater.

Assmann, H. (2002). Placer y ternura en educación: Hacia una sociedad aprendiente. Madrid: Narcea.

Becker E. (2003). La negación de la muerte. Barcelona: Editorial Kairós.

Castillo, I. y Castillo, R. (2013). La mediación biopedagógica desde una perspectiva ética. Revista Electrónica Educare, 17(2), 111-121. doi: http://doi.org/10.15359/ree.17-2.7

Díaz, F. y Hernández, A. (2005). Estrategias docentes para un aprendizaje significativo. Una interpretación constructivista. México: McGraw-Hill.

Fermozo, P. (1991). Teoría de la educación. Mexico. Trillas.

Freire, P. (2011). La educación como práctica de la libertad. Buenos Aires: Siglo XXI.

Gutiérrez, F. (2004). Pedagogía del aprendizaje. Guatemala: USAC.

Gutiérrez, F. y Prado, C. (2015). Ecopedagogía y ciudadanía planetaria (3a ed.). San José, Costa Rica: De La Salle Ediciones.

Gutiérrez, F. y Prieto, D. (1999). La mediación pedagógica: Apuntes para una educación a distancia alternativa (6 a ed.). Buenos Aires: CICCUS, La Crujía. 
doi: http://doi.org/10.15359/ree.24-1.21

URL: http://www.una.ac.cr/educare

CORREO: educare@una.cr

Hathaway, M. y Boff, L. (2014). El tao de la liberación: Una ecología de la transformación. Madrid: Trotta.

Hinkelammert, F. J. y Mora, H. (2012). Hacia una economía para la vida. Cartago, Costa Rica: Editorial Tecnológica de Costa Rica.

Jung, C. G. (1991). El problema del mal en la actualidad. En C. Zwei, y J. Abrams (Eds.), Encuentro con la sombra. El poder del lado oculto de la naturaleza humana (pp. 241-246). Barcelona. Kairós. Recuperado de https://www.josepmariacarbo.cat/themes/demo/assets/docs/ JUNG-CARL-Encuentro-con-la-sombra.pdf

Labarrere, A. F. (2003). Pensamiento: El análisis y la autorregulación en la actividad cognoscitiva de los estudiantes. México: Ángeles Editores.

Labarrere, A. F. (2006). Aprendizaje, complejidad y desarrollo: Agenda curricular para enseñar en los tiempos actuales. Revista de Psicologia, 15(2), 65-76. doi: https://doi.org/10.5354/0719$\underline{0581.2006 .17147}$

Maturana H. (1995). Realidad: ¿Objetiva o construida? Guadalajara: Instituto Tecnológico y de Estudios Superiores de Occidente ITESO.

Maturana, H. (2002). Transformación en la convivencia. Santiago: Dolmen.

Najmanovich D. (2005). Estética del pensamiento complejo. Andamios, Revista de Investigación Social, 1(2), 19-42. Recuperado de http://www.scielo.org.mx/pdf/anda/v1n2/v1n2a2.pdf

Najmanovich, D. (2008). Mirar con nuevos ojos. Nuevos paradigmas en la ciencia y pensamiento complejo. Buenos Aires: Biblos.

Pérez, R. Á. (2009). El constructivismo en los espacios educativos. Cartago, Costa Rica: CECC.

Pérez, R. Á.., Molina, Z., Hernández, Á., Rojas, G. y Murillo, E. (1991). Los procesos de enseñanza y aprendizaje en una sociedad democrática. San José, Costa Rica: MEP.

Prieto, D. (2017). Construirse para educar. Caminos de la educomunicación. Chasqui. Revista Latinoamericana de Comunicación, 135, 17-32. Recuperado en https://revistachasqui.org/ index.php/chasqui/article/view/3328

Sheldrake R. (1981). Una nueva ciencia de la vida. La hipótesis de la causación formativa. Barcelona: Editorial Kairós.

Tébar, L. (2009). El profesor mediador del aprendizaje. Bogota, Colombia: Magisterio Editorial.

Villarruel M. (2009). La práctica educativa del maestro mediador. Revista lberoamericana de educación, 50(3), 1-12. Recuperado de http://www.rieoei.org/deloslectores/2957Fuentes.pdf 